\title{
Supplement Table 1. Primers
}

\begin{tabular}{|c|c|c|c|}
\hline Gene & $\begin{array}{l}\text { Forward primer } \\
\left(5^{\prime}-3^{\prime}\right)\end{array}$ & $\begin{array}{l}\text { Reverse primer } \\
\left(5^{\prime}-3^{\prime}\right)\end{array}$ & $\begin{array}{l}\text { Length } \\
\text { (bases) }\end{array}$ \\
\hline \multicolumn{4}{|l|}{ Human } \\
\hline CASR & ccctctacgattgctgtggt & agtctgctggaggaggcata & 20 \\
\hline TNF- $\alpha$ & tcagcctcttctccttcctg & gccagagggctgattagaga & 20 \\
\hline IL1 $\beta$ & tccagggacaggatatggag & tctttcaacacgcaggacag & 20 \\
\hline IL6 & tacccccaggagaagattcc & agtgcctctttgctgctttc & 20 \\
\hline IL10 & ttacctggaggaggtgatgc & ggccttgctcttgttttcac & 20 \\
\hline IL 2 & gcaactcctgtcttgcattg & tgagcatcctggtgagtttg & 20 \\
\hline GAPDH & tcaccagggctgcttttaac & gacaagcttcccgttctcag & 20 \\
\hline \multicolumn{4}{|l|}{ Mouse } \\
\hline GAPDH & aactttggcattgtggaagg & ggatgcagggatgatgttct & 20 \\
\hline TNF- $\alpha$ & ccccaaagggatgagaagtt & cacttggtggtttgctacga & 20 \\
\hline IL1 $\beta$ & ggatgaggacatgagcacct & agctcatatgggtccgacag & 20 \\
\hline IL6 & ccggagaggagacttcacag & cagaattgccattgcacaac & 20 \\
\hline IL10 & gccttatcggaaatgatcca & aggggagaaatcgatgacag & 20 \\
\hline IL $17 \mathrm{~A}$ & ccagggagagcttcatctgt & aggaagtccttggcctcagt & 20 \\
\hline IFN- $\gamma$ & gctcttcctcatggctgttt & gtcaccatccttttgccagt & 20 \\
\hline
\end{tabular}

